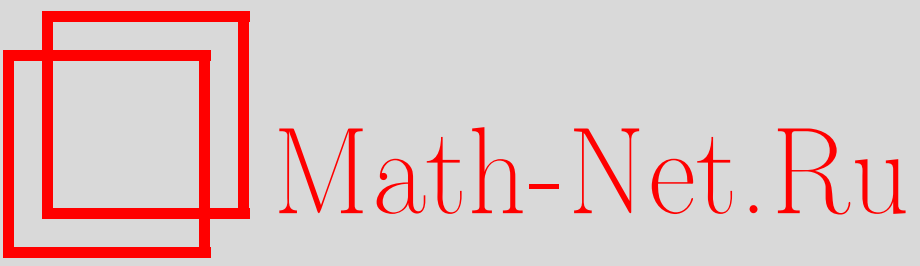

Д. Е. Бурланков, А. М. Самочадин, Потоки энергии гравитационных волн в жидкой сфере, ТМФ, 1999, том 120, номер 2, 342-351

DOI: https://doi.org/10.4213/tmf781

Использование Общероссийского математического портала Math-Net.Ru подразумевает, что вы прочитали и согласны с пользовательским соглашением

http://www.mathnet.ru/rus/agreement

Параметры загрузки:

IP: 34.229 .45 .116

26 апреля 2023 г., 10:09:06 


\author{
ТЕОРЕТИЧЕСКАЯ \\ И МАТЕМАТИЧЕСКАЯ \\ ФИЗИКА \\ Том 120, № 2 \\ август, 1999
}

(C) 1999 г.

Д.Е. Бурланков* , А.М. Самочадин*

\title{
ПОТОКИ ЭНЕРГИИ ГРАВИТАЦИОННЫХ ВОЛН В ЖИДКОЙ СФЕРЕ
}

Рассмотрена проблема переноса энергии на статическом фоновом пространствевремени, создаваемом жидким сферическим телом. Выведен квадратичный по возмущениям лагранжиан гравитации и жидкости в сопутствующей системе отсчета. Наложение калибровочных условий и интегрирование по угловым координатам производится на уровне интеграла действия, и поэтому задача оказывается двумерной. Выведены поток и плотность энергии возмущений. Рассмотрены различные варианты выбора калибровки. Выполнение закона сохранения энергии обеспечивается статичностью метрики и обращением в нуль лагранжевых вариаций.

\section{1. ВВЕДЕНИЕ}

В задаче описания бесконечно малых гравитационно-акустических колебаний жидкой сферы [1-3], несмотря на достаточно длинную историю, которую, по-видимому, нужно вести от работы Уилера и Редже [4], имеется еше немало принципиальных вопросов: например, проблемы выбора калибровки и описания переноса энергии гравитационно-акустическими колебаниями. Торн с сотрудниками [1] выбрали калибровку, в которой ненулевыми являются возмушения метрики, раскладываемые по обычным (скалярным) сферическим функциям. При этом уравнения Эйнштейна выглядят относительно просто. Достаточно сложной составляюшей этих уравнений оказываются уравнения динамики жидкости. В работе [3] использовались калибровочные степени свободы для диагонализации тензора возмушений метрики (рассматривались только четные моды), что упрошает вывод уравнений, однако учет уравнений динамики также приводит к довольно сложной системе дифференциальных уравнений пятого порядка.

Из физических соображений следует, что для внутренней задачи наиболее адекватной оказывается сопутствуюшая система для возмушений, где обрашены в нуль компоненты $h_{01}, h_{02}, h_{03}$. Для этого используются три калибровочных преобразования из четырех. Преобразование времени вывело бы из сопутствуюшей системы. Поэтому используемое нами исходное количество компонент тензора возмушений больше, чем у предыдущих авторов. При этом из уравнений выпадает вектор скорости, и выполнение гидродинамических уравнений гарантируется в соответствии с теоремой Гильберта [5]

\footnotetext{
*Нижегородский государственный университет, Нижний Новгород, Россия
} 
выполнением уравнений Эйнштейна. В итоге система описывается четырьмя дифференциальными уравнениями первого порядка, что соответствует распространению связанных акустической и гравитационной (четной) мод.

В качестве фона мы выберем пространственно конформно-плоскую метрику

$$
d s^{2}=e^{2 \nu} d t^{2}-e^{2 \mu-2 \nu}\left(d r^{2}+r^{2}\left(d \vartheta^{2}+\sin ^{2} \vartheta d \varphi^{2}\right)\right),
$$

удобную тем, что в ней найдена серия аналитических решений [6-8]. В работе [9] выведено разложение чисто гравитационного лагранжиана до второго порядка по возмушениям метрики

$$
\begin{aligned}
\delta_{2}\left(\sqrt{g} g^{i j} R_{i j}\right)= & \sqrt{g} g^{i j}\left(\delta \Gamma_{i l}^{k} \delta \Gamma_{j k}^{l}-\delta \Gamma_{i j}^{l} \delta \Gamma_{l k}^{k}\right)+ \\
& +\sqrt{g} R_{j}^{k}\left(h_{s}^{j} h_{k}^{s}-\frac{1}{2} h_{k}^{j} h_{s}^{s}-\frac{1}{4} \delta_{k}^{j}\left(h_{m}^{s} h_{s}^{m}-\frac{1}{2} h_{s}^{s} h_{m}^{m}\right)\right)
\end{aligned}
$$

где

$$
\delta \Gamma_{j k}^{i}=\frac{g^{i s}}{2}\left(\nabla_{j} h_{s k}+\nabla_{k} h_{j s}-\nabla_{s} h_{j k}\right) .
$$

В вакууме компоненты $R_{j}^{i}$ фона равняются нулю. В жидкости же они определяются через давление и плотность энергии соотношениями

$$
R_{0}^{0}=\frac{\varepsilon+3 p}{2}, \quad R_{1}^{1}=R_{2}^{2}=R_{3}^{3}=\frac{p-\varepsilon}{2} .
$$

\section{2. ЛАГРАНЖИАН ЖИДКОСТИ}

Как было показано в статье [10], действие для паскалевой жидкости определяется давлением, зависящим от энтальпии $\mu$,

$$
S_{f}=\int \sqrt{g} p(\mu) d^{4} x
$$

где $\mu=\sqrt{g^{i j} \sigma,_{i} \sigma, j}$. Вариация действия по переменной $\sigma$ приводит к уравнению

$$
\partial_{i}\left(\sqrt{g} \frac{\partial p}{\partial \sigma_{i}}\right)=\partial_{i}\left(\sqrt{g} \frac{d p}{d \mu} \frac{g^{i j} \sigma_{j}}{\mu}\right)=0
$$

которое является уравнением непрерывности в гидроаэродинамике, если величину

$$
\frac{g^{i j} \sigma_{j}}{\mu} \equiv u^{i}
$$

считать четырехвектором скорости (т.к. $g_{i j} u^{i} u^{j}=1$ ), а $d p / d \mu \equiv \rho$-плотностью вещества. Сопутствуюшая система отсчета - это система, где $\sigma$ определяется только переменной $t: \sigma=t$. Тогда $\mu=\sqrt{g^{00}}$ есть термодинамическая функция жидкости, однозначно 
связываемая с метрическим тензором. Вариация действия по компоненте $g^{00}$ определяет плотность энергии

$$
\varepsilon=\mu \frac{d p}{d \mu}-p
$$

Локальная скорость звука $а$ определяется соотношением

$$
\frac{c^{2}}{a^{2}} \equiv q=\frac{d \varepsilon}{d p}
$$

и может быть найдена по фоновому решению $q=\varepsilon^{\prime} / p^{\prime}$.

При рассмотрении возмущений на стационарном фоне мы должны разложить давление по возмущениям до второго порядка включительно, т.е.

$$
\widetilde{p}=p+\frac{d p}{d \mu}\left(\delta_{1} \mu+\delta_{2} \mu\right)+\frac{d^{2} p}{d \mu^{2}} \frac{\delta_{1} \mu^{2}}{2} .
$$

Учитывая, что

$$
\frac{d^{2} p}{d \mu^{2}}=\frac{d \rho}{d \mu}=\frac{d}{d \mu} \frac{\varepsilon+p}{\mu}=q \frac{\varepsilon+p}{\mu^{2}}
$$

имеем

$$
\begin{gathered}
\widetilde{p}=p+(\varepsilon+p)\left(\frac{\delta_{1} \mu}{\mu}+q \frac{\delta_{1} \mu^{2}}{2}\right) \\
\sqrt{\widetilde{g}} \approx \sqrt{g}\left(1+\frac{h_{i}^{i}}{2}-\frac{1}{4}\left(h_{j}^{i} h_{i}^{j}-\frac{\left(h_{i}^{i}\right)^{2}}{2}\right)\right) .
\end{gathered}
$$

Возмушения $\mu$ (если положить $\sigma=1+\eta$ ) можно записать в виде

$$
\begin{aligned}
\widetilde{\mu} & =\sqrt{\left(g^{i j}-h^{i j}+h_{k}^{i} h^{k j}\right)\left(\delta_{i}^{0}+\eta_{i}\right)\left(\delta_{j}^{0}+\eta_{j}\right)}= \\
& =\mu\left(1-\frac{1}{2} h_{0}^{0}+\eta, 0-\frac{1}{8}\left(h_{0}^{0}-2 \eta, 0\right)^{2}-h_{0}^{i} \eta,{ }_{i}+\frac{1}{2} h_{k}^{0} h_{0}^{k}+\frac{g^{i j}}{2 g^{00}} \eta,_{i} \eta,{ }_{j}\right) .
\end{aligned}
$$

Уравнение непрерывности в первом порядке малости

$$
\begin{aligned}
\partial_{i}( & \left.\sqrt{g}\left(1+\frac{1}{2} h_{s}^{s}\right) \rho\left(1+q \frac{\delta \mu}{\mu}\right) \frac{g^{i j}\left(\delta_{j}^{0}+\eta, j\right)}{\mu+\delta \mu}\right) \approx \\
& \approx \partial_{0}\left(\sqrt{g} \rho\left(\frac{1}{2} h_{s}^{s}+(q-1) \frac{\delta \mu}{\mu}\right)\right)+\partial_{i}\left(\sqrt{g} \frac{\rho}{\mu} g^{i j} \eta,{ }_{j}\right)=0
\end{aligned}
$$

оказывается дифференциальным уравнением второго порядка относительно возмушения $\eta$. Это уравнение присоединяется к уравнениям Эйнштейна, в правые части которых добавляются члены, содержащие четырехвектор скорости.

Однако можно распорядиться калибровочным преобразованием времени не для уничтожения какой-то компоненты возмущения метрики, а для уничтожения величины $\eta$, что 
оставляет возмушенную систему координат сопутствующей. Оставшиеся три калибровочные функции можно использовать для избавления от компонент $h_{01}, h_{02}, h_{03}$. Возмущенный тензор энергии-импульса $T_{j}^{i}$ при этом по-прежнему диагонален и имеет вид

$$
T_{0}^{0}=\varepsilon+\delta \varepsilon, \quad T_{1}^{1}=T_{2}^{2}=T_{3}^{3}=-p-\delta p .
$$

Решать теперь нужно только уравнения Эйнштейна.

Разложение до второго порядка лагранжиана жидкости (индексы $i, j, k$ меняются от 1 до 3, т.к. индекс 0 мы выделяем явно) принимает вид

$$
\delta_{2}(\sqrt{g} p)=\frac{p}{8}\left(2 h_{0}^{0} h_{i}^{i}+\left(h_{i}^{i}\right)^{2}-\left(h_{0}^{0}\right)^{2}-2 h_{j}^{i} h_{i}^{j}\right)-\frac{\varepsilon+p}{8}\left(2 h_{0}^{0} h_{i}^{i}+(q+1)\left(h_{0}^{0}\right)^{2}\right) .
$$

Полное действие для самогравитируюшей жидкой сферы есть $S=S_{g}+S_{f}$. Учитывая выражение (2), получаем полный лагранжиан системы

$$
\begin{aligned}
\mathcal{L}= & -\frac{c^{4}}{16 \pi k} \sqrt{g} g^{i j}\left(\delta \Gamma_{i l}^{k} \delta \Gamma_{j k}^{l}-\delta \Gamma_{i j}^{l} \delta \Gamma_{l k}^{k}\right)+ \\
& +\frac{\sqrt{g}}{16}\left[((2 q-1) \varepsilon+(2 q-3) p)\left(h_{0}^{0}\right)^{2}+(\varepsilon-p)\left(2 h_{j}^{i} h_{i}^{j}-2 h_{0}^{0} h_{i}^{i}-\left(h_{i}^{i}\right)^{2}\right)\right] .
\end{aligned}
$$

\section{3. РАЗЛОЖЕНИЕ ПО СФЕРИЧЕСКИМ ФУНКЦИЯМ}

При преобразовании угловых переменных компоненты, у которых отсутствуют индексы $\theta$ и $\phi$, преобразуются как скаляры и раскладываются по обычным сферическим функциям. Преобразованием врашения любую такую функцию можно привести к функции $Y_{l m}=Y_{l 0}$, зависящей только от угла $\theta$ и пропорциональной полиному Лежандра от $\cos \theta$. Эти функции мы будем называть $Y_{l}$.

Четные компоненты $h_{0 \alpha}$ и $h_{1 \alpha}(\alpha=2,3)$ при преобразованиях $\theta$ и $\phi$ ведут себя как векторы и раскладываются по векторным функциям $Y_{v}=\partial_{\theta} Y_{l}$ (первым присоединенным полиномам Лежандра от $\cos \theta)$, а компоненты $h_{\alpha \beta}$ раскладываются как по скалярным, так и по тензорным (вторым присоединенным полиномам Лежандра) функциям $Y_{t}$. Обозначив

$$
l_{1}=\frac{l(l+1)}{2}, \quad l_{2}=l_{1}-1=\frac{(l-1)(l+2)}{2},
$$

можно записать следующие необходимые для вычислений дифференциальные соотношения между используемыми сферическими функциями:

$$
\partial_{\theta} Y_{l}=Y_{v}, \quad \partial_{\theta} Y_{v}=-Y_{t}-l_{1} Y_{l}, \quad \partial_{\theta} Y_{t}=l_{2} Y_{v}-2(\operatorname{ctg} \theta) Y_{t} .
$$

Для четных возмущений имеем

$$
\begin{aligned}
& h_{00}=Y_{l} a_{00} e^{2 \nu}, \quad h_{01}=Y_{l} a_{01}, \quad h_{02}=Y_{v} r a_{02} \text {, } \\
& h_{11}=Y_{l} a_{11} e^{2 \nu}, \quad h_{12}=Y_{v} r e^{-2 \nu} a_{12} \text {, } \\
& h_{22}=\left(Y_{l} a_{22}+Y_{t} a_{33}\right) r^{2}, \quad h_{33}=\left(Y_{l} a_{22}-Y_{t} a_{33}\right) r^{2} \sin ^{2} \theta \text {, }
\end{aligned}
$$

для нечетных -

$$
h_{03}=Y_{v} a_{03} r \sin \theta, \quad h_{13}=Y_{v} a_{13} e^{-2 \nu} r \sin \theta, \quad h_{23}=Y_{t} a_{23} r^{2} \sin \theta .
$$




\section{4. УРАВНЕНИЯ ДВИЖЕНИЯ}

Уравнения для малых возмушений в общем случае приведены в статье [4] и имеют вид

$$
\delta R_{i j}=\nabla_{k} \delta \Gamma_{i j}^{k}-\nabla_{j} \delta \Gamma_{i k}^{k} .
$$

Так как фоновое пространство не плоское, то ковариантные производные не перестановочны. Определяя вектор $a_{i}=\nabla_{s} h_{i}^{s}$, получим

$$
\delta R_{j}^{k}=\frac{1}{2}\left[g^{k i}\left(\nabla_{i} a_{j}+\nabla_{j} a_{i}-\Delta h_{i j}-\nabla_{i} \nabla_{j} h_{s}^{s}\right)+R_{i}^{k} h_{j}^{i}-R_{j}^{i} h_{i}^{k}\right]-R^{k i}{ }_{j s} h_{i}^{s} .
$$

Из тождеств Гильберта

$$
\widetilde{\nabla}_{i}\left(G_{j}^{i}+\delta G_{j}^{i}\right) \approx\left(\nabla_{i} G_{j}^{i}\right)+\left(\nabla_{i} \delta G_{j}^{i}+\delta \Gamma_{j s}^{i} G_{i}^{s}-\delta \Gamma_{i j}^{s} G_{s}^{i}\right)=0
$$

для возмушений следует связь

$$
\nabla_{i} \delta G_{j}^{i}+\delta \Gamma_{i s}^{i} G_{j}^{s}-\delta \Gamma_{i j}^{s} G_{s}^{i}=0
$$

которая при $j=\vartheta$ дает

$$
\delta p=-\frac{\varepsilon+p}{2} h_{0}^{0}
$$

а при $j=0-$

$$
\delta \varepsilon=-\frac{\varepsilon+p}{2}\left(h_{1}^{1}+h_{2}^{2}+h_{3}^{3}\right) .
$$

Поскольку $\delta \varepsilon=q \delta p$, то получается алгебраическая связь компонент тензора возмущений

$$
h_{1}^{1}+h_{2}^{2}+h_{3}^{3}=q h_{0}^{0} .
$$

Тогда уравнения (5) приводятся к виду

$$
\begin{aligned}
\frac{c^{4}}{16 \pi k} \delta R_{0}^{0}+\frac{q+3}{2} \frac{\varepsilon+p}{2} h_{0}^{0} & =0, \\
\frac{c^{4}}{16 \pi k} \delta R_{j}^{i}+\delta_{j}^{i} \frac{1-q}{2} \frac{\varepsilon+p}{2} h_{0}^{0} & =0, \quad i \neq j, \\
\delta R_{j}^{0} & =0 .
\end{aligned}
$$

После разложения по сферическим функциям и фурье-гармоникам относительно временной переменной эти уравнения записываются в виде системы четырех обыкновенных дифференциальных уравнений для функций только от радиуса. Если ввести обозначения

$$
\mathcal{B}=1+r\left(\mu^{\prime}-\nu^{\prime}\right), \quad \mathcal{C}=3 \mathcal{B}-r \nu^{\prime}, \quad \mathcal{D}=\mathcal{B}^{2}-1,
$$

то данная система после выполнения соотношения (7)

$$
a_{11}=-\left(q a_{00}+2 a_{22}\right)
$$


принимает вид

$$
\begin{aligned}
a_{12}^{\prime}= & -\frac{1}{r}\left(q a_{00}+\mathcal{C} a_{12}+a_{22}+l_{2} a_{33}\right), \\
a_{22}^{\prime}= & -\frac{1}{r}\left(q a_{00} \mathcal{B}+l_{1} a_{12}+\mathcal{C} a_{22}\right), \\
a_{00}^{\prime}= & -\frac{1}{r \mathcal{B}}\left[-\left(r \nu^{\prime}\right)^{2} a_{22}+r \nu^{\prime}\left(q a_{00} \mathcal{B}-l_{1} a_{12}+2 \mathcal{B} a_{22}\right)+\right. \\
& +\left(q \mathcal{D}-l_{1}\right) a_{00}-l_{1} \mathcal{B} a_{12}+\left(3 \mathcal{D}+l_{1}\right) a_{22}-l_{1} l_{2} a_{33}+ \\
& \left.+\frac{r^{2}}{2} e^{2 \mu-2 \nu}\left((\varepsilon-(2 q-1) p) a_{00}-4 p a_{22}\right)-e^{2 \mu-4 \nu}(r \omega)^{2} a_{22}\right], \\
a_{33}^{\prime}= & \frac{1}{r l_{2} \mathcal{B}}\left[-\left(r \nu^{\prime}\right)^{2} a_{22}+r \nu^{\prime}\left(3 \mathcal{B}\left(a_{00}-a_{22}\right)-l_{1} a_{12}\right)+\right. \\
& +\left(3 \mathcal{D}-l_{2}\right) a_{00}-2 l_{2} \mathcal{B} a_{12}-\left(2 \mathcal{D}-l_{2}\right) a_{22}-l_{1} l_{2} a_{33}+ \\
& \left.+\frac{r^{2}}{2} e^{2 \mu-2 \nu}\left((\varepsilon-3 p) a_{00}+4 p a_{22}\right)+e^{2 \mu-4 \nu}(r \omega)^{2}\left(\mathcal{B} a_{12}-a_{22}\right)\right] .
\end{aligned}
$$

Для нечетных мод

$$
\begin{aligned}
& a_{13}^{\prime}=-\frac{1}{r}\left(\mathcal{C} a_{13}+l_{2} a_{23}\right), \\
& a_{23}^{\prime}=\frac{1}{r l_{2}}\left(e^{2 \mu-4 \nu}(r \omega)^{2}-2 l_{2}\right) a_{13} .
\end{aligned}
$$

4.1. Асимптотика в центре. При $r \rightarrow 0$ имеем $\mathcal{B} \rightarrow 1, \mathcal{C} \rightarrow 3, \mathcal{D} \rightarrow 0$ и система уравнений (11)-(14) принимает вид

$$
\begin{aligned}
& r a_{00}^{\prime}=l_{1}\left(a_{00}+a_{12}-a_{22}+l_{2} a_{33}\right), \\
& r a_{12}^{\prime}=-\left(q a_{00}+3 a_{12}+a_{22}+l_{2} a_{33}\right), \\
& r a_{22}^{\prime}=-\left(3 q a_{00}+l_{1} a_{12}+3 a_{22}\right), \\
& r a_{33}^{\prime}=-\left(a_{00}+2 a_{12}-a_{22}+l_{1} a_{33}\right) .
\end{aligned}
$$

Эта система имеет решения вида $r^{k}$, где число $k$ может принимать четыре значения:

$$
k=\{-(l+3),-(l+1), l, l-2\}
$$

из которых два являются приемлемыми (при $l=0,1$ - только одно). Для нечетных мод

$$
\begin{aligned}
& r a_{13}^{\prime}=-\left(3 a_{13}+l_{2} a_{23}\right), \\
& r a_{23}^{\prime}=-2 a_{13}
\end{aligned}
$$

и $k$ принимает значения $k=\{l-1,-(l+2)\}$. Несингулярным в центре является одно решение. 
4.2. Моды с $l=0$ и $l=1$. Отдельного рассмотрения требуют моды с $l=0$ (радиальные колебания) и $l=1$ (дипольные колебания), в которых отсутствуют гравитационные компоненты и колебания являются чисто акустическими.

В случае радиальных колебаний из уравнений выпадают переменные $a_{12}$ и $a_{33}$, так что остается система двух связанных дифференциальных уравнений первого порядка, описывающая радиальные акустические колебания,

$$
\begin{aligned}
& a_{00}^{\prime}=-\frac{1}{r \mathcal{B}}\left(\mathcal{B} q r \nu^{\prime}-e^{2 \mu-2 \nu} r^{2} \frac{\varepsilon+p}{2}\right) a_{00}+4 r \nu^{\prime} a_{22}, \\
& a_{22}^{\prime}=-\frac{1}{r}\left(\mathcal{B} q a_{00}+\mathcal{C} a_{22}\right) .
\end{aligned}
$$

При $l=1$ из уравнений выпадает переменная $a_{33}$, но из условия $a_{33}^{\prime}=0$ можно выразить $a_{12}$ через $a_{00}$ и $a_{22}$, так что опять получается система только двух дифференциальных уравнений первого порядка. Вводя переменные

$$
u=r^{3} e^{3 \mu-4 \nu} \frac{a_{22}+a_{12}}{2}, \quad v=r^{3} e^{3 \mu-4 \nu} \frac{a_{22}-a_{12}}{2}, \quad w=r^{3} e^{3 \mu-4 \nu} \frac{a_{00}}{2},
$$

получаем для них систему дифференциальных уравнений

$$
\begin{aligned}
r u^{\prime} & =-u-q w\left(2+r\left(\mu^{\prime}-\nu^{\prime}\right)\right), \\
r v^{\prime} & =v-q w r\left(\mu^{\prime}-\nu^{\prime}\right), \\
w & =-\frac{\left(r \nu^{\prime}\left(B-r \nu^{\prime}\right)-(r \omega)^{2} e^{2 \mu-4 \nu}\right)(u+v)+\left(\mathcal{B}(r \omega)^{2} e^{2 \mu-4 \nu}-r \nu^{\prime}\right)(u-v)}{3 \mathcal{D}+\varepsilon e^{2 \mu-2 \nu}} .
\end{aligned}
$$

Здесь содержится только одна мода - акустическая.

4.3. Сшивание со внешним решением. На поверхности звезды выполняются соотношения $p=0$ и $\delta p=0$, что приводит к условию на границе $h_{00}=0$. При заданной частоте $\omega$ два независимых в центре решения $r^{l}$ и $r^{l-2}$ на поверхности принимают значения $h_{00}^{(1)}$ и $h_{00}^{(2)}$ и требование $h_{00}=0$ определяет относительные амплитуды этих мод.

Эти решения должны сшиваться со внешними решениями [11], для чего нужно провести соответствуюшее калибровочное преобразование.

Для нечетных мод ситуация упрошается. Уравнения (15), (16) не содержат параметров, испытывающих скачок на границе тела (например, $\varepsilon$ ), поэтому отсутствует их отражение от границы, и единственная несингулярная в центре ветвь непрерывно продолжается до бесконечности уже вне тела.

\section{5. ЛАГРАНЖИАН, ПЛОТНОСТЬ И ПОТОК ЭНЕРГИИ МАЛЫХ КОЛЕБАНИЙ}

Как и в случае пространства Шварцшильда [9], лагранжиан квадратичен по возмущениям и может быть проинтегрирован по угловым переменным с учетом соотношений

$$
\begin{gathered}
\left\langle Y_{v}^{2}\right\rangle=2 l_{1}, \quad\left\langle Y_{t}^{2}\right\rangle=l_{1} l_{2}, \\
\left\langle\left(\frac{Y_{t}}{\sin \theta}\right)^{2}\right\rangle=\frac{2 l+1}{4} l_{1} l_{2}, \quad\left\langle\left(\frac{Y_{v}}{\sin \theta}\right)^{2}\right\rangle=l_{1}(2 l+1), \\
\left\langle Y_{l} Y_{t}\right\rangle=\frac{l(l-1)}{2}, \quad\left\langle Y_{l} Y_{v} \operatorname{ctg} \theta\right\rangle=-l, \quad\left\langle Y_{v} Y_{t} \operatorname{ctg} \theta\right\rangle=l_{1}(l-1),
\end{gathered}
$$


где

$$
\langle A\rangle=\frac{\int_{-1}^{1} A \sin \theta d \theta}{\int_{-1}^{1} Y_{l}^{2} \sin \theta d \theta} .
$$

После интегрирования $\left(\mathrm{c} \sqrt{g}\right.$, так что $\left.S=\int \mathcal{L} d r d t\right)$ лагранжиан четных мод (4) зависит от функций двух переменных и принимает вид

$$
\begin{aligned}
\mathcal{L}_{e}= & \frac{c^{4}}{4 k(2 l+1)}\left[e^{3 \mu-4 \nu} r^{2}\left(-2 \dot{a}_{11} \dot{a}_{22}+2 l_{1} \dot{a}_{12}^{2}-\dot{a}_{22}^{2}+l_{1} l_{2} \dot{a}_{33}^{2}\right)+\right. \\
& +e^{\mu}\left(r^{2}\left(-2 a_{00}^{\prime} a_{22}^{\prime}+a_{22}^{\prime 2}-l_{1} l_{2} a_{33}^{\prime 2}\right)+2 l_{1} r\left(\left(a_{11}^{\prime}-a_{00}^{\prime}\right) a_{12}+\right.\right. \\
& \left.+a_{12}^{\prime}\left(a_{00}+a_{11}-2 a_{22}\right)-2 l_{2} a_{33}^{\prime} a_{12}\right)+ \\
& +2 l_{1}\left(a_{00}\left(l_{2} a_{33}-a_{11}-a_{22}\right)+a_{11}\left(a_{22}-l_{2} a_{33}\right)-l_{2} a_{33}^{2}\right)- \\
& -r^{2} \nu^{\prime}\left(2 a_{22}^{\prime}+a_{00}^{\prime}+a_{11}^{\prime}\right)\left(a_{00}+a_{11}\right)+ \\
& +2 r \mathcal{B}\left(\left(a_{00}^{\prime}-a_{11}^{\prime}\right)\left(a_{11}-a_{22}\right)-4 l_{1} a_{12}^{\prime} a_{12}-2 l_{1} l_{2} a_{33}^{\prime} a_{33}\right)- \\
& \left.\left.-2 l_{1} r \nu^{\prime} a_{12}\left(a_{00}+a_{11}+2 a_{22}\right)+2 l_{1} \mathcal{B} a_{12}\left(3 a_{00}-a_{11}-2 a_{22}\right)\right)\right]+ \\
& +\frac{4 \pi}{2 l+1} e^{3 \mu-2 \nu} r^{2}\left[(\varepsilon+p) a_{00}^{2} \frac{q-1}{2}+\right. \\
& \left.+(\varepsilon-p)\left(\frac{\left(a_{00}+a_{11}\right)^{2}}{4}+a_{22}\left(a_{00}-a_{11}\right)+2 a_{12}^{2} l_{1}+l_{1} l_{2} a_{33}^{2}\right)\right] .
\end{aligned}
$$

Плотность энергии четных мод

$$
\mathcal{W}=\frac{\partial \mathcal{L}}{\partial \dot{a}_{00}} \dot{a}_{00}+\frac{\partial \mathcal{L}}{\partial \dot{a}_{11}} \dot{a}_{11}+\frac{\partial \mathcal{L}}{\partial \dot{a}_{12}} \dot{a}_{12}+\frac{\partial \mathcal{L}}{\partial \dot{a}_{22}} \dot{a}_{22}+\frac{\partial \mathcal{L}}{\partial \dot{a}_{33}} \dot{a}_{33}-\mathcal{L} .
$$

Так как лагранжиан квадратичен, то плотность $\mathcal{W}$ совпадает по структуре с лагранжианом при уничтожении членов, линейных по $\dot{a}_{i j}$ (в данном лагранжиане их нет), и при смене знака у членов без производных по времени. Поток энергии

$$
\begin{aligned}
\mathcal{U}=\frac{\partial \mathcal{L}}{\partial a_{00}^{\prime}} \dot{a}_{00}+\frac{\partial \mathcal{L}}{\partial a_{11}^{\prime}} \dot{a}_{11}+\frac{\partial \mathcal{L}}{\partial a_{12}^{\prime}} \dot{a}_{12}+\frac{\partial \mathcal{L}}{\partial a_{22}^{\prime}} \dot{a}_{22}+\frac{\partial \mathcal{L}}{\partial a_{33}^{\prime}} \dot{a}_{33}= \\
=-\frac{c^{4}}{4 k(2 l+1)} e^{\mu}\left[2 r^{2}\left(\dot{a}_{22}\left(a_{00}^{\prime}-a_{22}^{\prime}\right)+l_{1} l_{2} \dot{a}_{33} a_{33}^{\prime}\right)+\right. \\
\\
+2 r l_{1}\left(\dot{a}_{12}\left(2 a_{22}-a_{00}-a_{11}\right)-\dot{a}_{11} a_{12}+2 l_{2} \dot{a}_{33} a_{12}\right)+ \\
\\
+2 r \mathcal{B}\left(\dot{a}_{11}\left(a_{11}-a_{22}\right)+4 l_{1} \dot{a}_{12} a_{12}+2 l_{1} l_{2} \dot{a}_{33} a_{33}\right)+ \\
\\
\left.+r^{2} \nu^{\prime}\left(\dot{a}_{11}+2 \dot{a}_{22}\right)\left(a_{00}+a_{11}\right)\right] .
\end{aligned}
$$

Вариационные производные

$$
\frac{\delta \mathcal{L}}{\delta h_{00}}=\frac{\partial \mathcal{L}}{\partial a_{00}}-\partial_{t} \frac{\partial \mathcal{L}}{\partial \dot{a}_{00}}-\partial_{r} \frac{\partial \mathcal{L}}{\partial a_{00}^{\prime}}, \quad \frac{\delta \mathcal{L}}{\delta h_{11}}, \quad \frac{\delta \mathcal{L}}{\delta h_{12}}, \quad \frac{\delta \mathcal{L}}{\delta h_{22}}, \quad \frac{\delta \mathcal{L}}{\delta h_{33}}
$$

в соответствии с уравнениями (10)-(14) обрашаются в нуль, что приводит к уравнению сохранения потока

$$
\partial_{t} \mathcal{W}+\mathcal{U}^{\prime}=0
$$


Аналогично записываются соответствуюшие соотношения для нечетных мод. Лагранжиан

$$
\begin{aligned}
\mathcal{L}= & \frac{c^{4}}{16 k(2 l+1)} l_{1}\left(e^{3 \mu-4 \nu} r^{2}\left(2 \dot{a}_{13}^{2}+l_{2} \dot{a}_{23}^{2}\right)-\right. \\
& -e^{\mu}\left(r^{2} a_{23}^{\prime 2} l_{2}+4 r \mathcal{B}\left(2 a_{13}^{\prime} a_{13}+l_{2} a_{23}^{\prime} a_{23}\right)+\right. \\
& \left.\left.+4 r l_{2} a_{23}^{\prime} a_{13}+4\left(l_{1} a_{13}^{2}+l_{2} a_{23}^{2}\right)\right)\right)+ \\
& +\frac{\pi}{2 l+1}(\varepsilon-p) l_{1}\left(2 a_{13}^{2}+l_{2} a_{23}^{2}\right)
\end{aligned}
$$

генерирует плотность

$$
\begin{aligned}
\mathcal{W}= & \frac{c^{4}}{16 k(2 l+1)} l_{1}\left(e^{3 \mu-4 \nu} r^{2}\left(2 \dot{a}_{13}^{2}+l_{2} \dot{a}_{23}^{2}\right)+\right. \\
& +e^{\mu}\left(l_{2} r^{2} a_{23}^{\prime 2}+4 r \mathcal{B}\left(2 a_{13}^{\prime} a_{13}+l_{2} a_{23}^{\prime} a_{23}\right)+\right. \\
& \left.\left.+4 r l_{2} a_{23}^{\prime} a_{13}+4\left(l_{1} a_{13}^{2}+l_{2} a_{23}^{2}\right)\right)\right)- \\
& -\frac{\pi}{2 l+1}(\varepsilon-p) l_{1}\left(2 a_{13}^{2}+l_{2} a_{23}^{2}\right)
\end{aligned}
$$

и поток энергии

$$
\mathcal{U}=-\frac{c^{4}}{2 k(2 l+1)} r e^{\mu} l_{1}\left(l_{2}\left(r a_{23}^{\prime}+2 a_{13}\right) \dot{a}_{23}+2 \mathcal{B}\left(2 a_{13} \dot{a}_{13}+l_{2} a_{23} \dot{a}_{23}\right)\right) .
$$

\section{6. ЗАКЛЮЧЕНИЕ}

Таким образом, в случае стационарного фона для бесконечно малых гравитационно-акустических возмушений плотность и поток энергии четко определены и выводятся из квадратичного по возмушениям лагранжиана, причем калибровочные условия можно накладывать прямо в лагранжиане.

На уровне лагранжиана выполняется также интегрирование по угловым переменным, что превращает данную задачу в двумерную.

Фоновое пространство-время для описания колебаний и потока энергии удобно выбирать в аналитическом виде. В статье [8] представлена серия аналитических решений с единственным параметром - отношением внешнего радиуса к гравитационному. Поверхность зафиксирована равенством нулю давления, при этом плотность энергии отлична от нуля. Каждое решение при соответствуюшем масштабном преобразовании давления и плотности энергии может описывать звезду произвольного внешнего радиуса.

Благодарности. Вычисления проводились в системе Reduce A. Hern'a на базе Lisp'a T. Yamamoto. Один из авторов (Д.Е.Б.) с большой благодарностью вспоминает совместное терпеливое и тщательное обсуждение данных проблем летом 1994 г. с С. Чандрасекаром. Он также благодарен Р. Валди за предоставленную тогда же возможность заниматься данными исследованиями в его лаборатории. Авторы признательны П. К. Бражнику за помощь в работе. 


\section{Список литературы}

[1] K.S. Thorn, A. Campolattoro. Astrophys. J. 1967. V. 149. P. 591-611.

[2] L. Lindblom, S. Detweiler. Astrophys. J. Suppl. 1983. V. 53. P. 73-92.

[3] S. Chandrasekhar, V. Ferrari. Proc. R. Soc. London. A. 1991. V. 432. P. 247-279.

[4] T. Regge, J. A. Wheeler. Phys. Rev. 1957. V. 108. P. 1063-1069.

[5] D. Hilbert. Nachrichten von der Kön. Ges. der Wiss. zu Göttingen. Math. -Phys. K1. 1915. № 3. P. 395-407.

[6] S. P. Goldman. Astrophys. J. 1978. V. 226. P. 1079-1084.

[7] H. Knutsen. Gen. Rel. Grav. 1991. V. 23. P. 843-859.

[8] Д. Е. Бурланков. ТМФ. 1993. Т. 95. № 1. С. 135-145.

[9] Д. Е. Бурланков, А. М. Самочадин. ТМФ. 1999. Т. 120. № 1. С. 168-176.

[10] B. F. Schutz. Phys. Rev. D. 1971. V. 4. P. 3559-3566.

[11] F. Zerilli. Phys. Rev. Lett. 1970. V. 24. P. 737-738.

Поступила в редакцию 24.XII.1998 г. 